\title{
THE CURRENT STAR FORMATION RATE OF THE LOCAL UNIVERSE
}

\author{
J. Gallego, ${ }^{1}$ J. Zamorano, ${ }^{1}$ A. Aragón-Salamanca, ${ }^{2}$ And M. Rego ${ }^{1}$ \\ Received 1995 July 26; accepted 1995 September 27
}

\begin{abstract}
The Universidad Complutense de Madrid (UCM) survey is a long-term project aimed at finding and analyzing star-forming galaxies detected by their $\mathrm{H} \alpha$ emission in Schmidt objective-prism plates. The instrumental setup limits the volume of the universe surveyed to a redshift $z \lesssim 0.045$. So far we have discovered several hundred emission-line galaxies (ELGs) undergoing star formation at a wide range of levels. Analyzing a complete sample of ELGs from the UCM survey, we have computed the $\mathrm{H} \alpha$ luminosity function for the star-forming galaxies in the surveyed volume of the universe. A Schechter function provides a good fit to the $\mathrm{H} \alpha$ luminosity function with the following parameters: $\alpha=-1.3 \pm 0.2, L^{*}(\mathrm{H} \alpha)=10^{42.15 \pm 0.08} \mathrm{ergs} \mathrm{s}^{-1}$, and $\phi^{*}=10^{-3.2 \pm 0.2} \mathrm{Mpc}^{-3}$ for $H_{0}=50$ $\mathrm{km} \mathrm{s}^{-1} \mathrm{Mpc}^{-1}$. Integrating over the full range of luminosities, we obtain an H $\alpha$ luminosity density of $10^{39.1 \pm 0.2}$ ergs $\mathrm{s}^{-1} \mathrm{Mpc}^{-3}$. Using the $\mathrm{H} \alpha$ emission as a star formation rate (SFR) estimator, this translates into a SFR density for the local universe of $0.013_{-0.005}^{+0.007} M_{\odot} \mathrm{yr}^{-1} \mathrm{Mpc}^{-3}$ in star-forming galaxies with $\mathrm{EW}(\mathrm{H} \alpha+[\mathrm{N}$ II] $)>10 \AA$ and $z \lesssim 0.045$ for a Scalo initial mass function. This is the first observational determination of this quantity, which will provide a direct test of current galaxy formation and evolution models.
\end{abstract}

Subject headings: galaxies: fundamental parameters - galaxies: luminosity function, mass function

\section{INTRODUCTION}

Studying the properties of star-forming galaxies at low redshift, and quantifying the amount and location of star formation in the local universe, are necessary steps toward understanding galaxy formation and evolution. They can provide a crucial test for galaxy formation and evolution models. Such models are now able to put together cosmology, dark matter, gas, and stars and make definite predictions for the properties of the present-day galaxy population and its evolution with redshift. In particular, they can predict current star formation rates (Kauffmann, White, \& Guiderdoni 1993; Cole et al. 1994), but very few observational constraints exist. Substantial data sets have been put together (e.g., Kennicutt 1992 and references therein), but not for a star formationselected sample of galaxies.

Moreover, extensive observational effort has been devoted to the study of the properties and evolution of high-redshift galaxies (e.g., deep counts, deep redshift surveys, and gravitational lensing), but one of the major problems that arises when analyzing high- $z$ data is how to make a meaningful comparison with local samples, usually obtained using very different observational techniques. Carrying out such a comparison involves two distinct steps: First, we need well-understood data for local samples that are directly related to the distant ones, and, second, we have to transform the apparent properties of the local objects to predict how they would look at high $z$ (i.e., introduce cosmological corrections and selection effects). If either or both of these steps are not accurate enough, apparent differences between local and distant samples could mimic evolution and lead to erroneous conclusions. A clear example where this problem is very important occurs in the interpretation of the so-called faint blue galaxies problem, where much of the recent controversy depends very much on what different authors believe about the properties of local galaxy samples,

\footnotetext{
${ }_{1}^{1}$ Departamento de Astrofísica, Universidad Complutense de Madrid E-28040 Madrid, Spain; jgm,jaz,mrf@ucmast.fis.ucm.es.

${ }^{2}$ Institute of Astronomy, University of Cambridge, Madingley Road, Cambridge CB3 0HA, England, UK; aas@ast.cam.ac.uk.
}

and, in particular, about the local galaxy luminosity function (LF) (see, e.g., Koo \& Kron 1992 and Broadhurst, Ellis, \& Glazebrook 1992 for different views on this problem).

Closely related to this is the recent evidence found in deep galaxy redshift surveys selected in the optical and in the near-IR, which suggests that star formation activity substantially increases with redshift from $z \simeq 0$ to $z \simeq 1$ (Songaila et al. 1994; Colless 1995; Ellis et al. 1995; Lilly et al. 1995). Although the causes of this increase in star formation are not yet clear (interactions and mergers have been suggested), it could explain the faint blue galaxy excess and the suggested evolution in the optical field LF (see Aragón-Salamanca 1995 for a recent review). Paradoxically, we seem to know more about the numbers of star-forming galaxies at intermediate and high redshifts than locally. This Letter tackles this problem directly, providing the first published estimate of the numbers of nearby star-forming galaxies and the star formation rate (SFR) density of the local universe.

The $\mathrm{H} \alpha$ luminosity is a very good direct measurement of the current SFR, since it is directly related to the number of massive stars (see, e.g., Kennicutt 1992; Gallagher \& Gibson 1993). It is better than other optical Balmer lines such as $\mathrm{H} \beta$ or $\mathrm{H} \gamma$, which are affected more by stellar absorption and reddening. Metallic nebular lines like [O III] $\lambda 5007,[\mathrm{O}$ II] $\lambda 3727$ (affected by excitation and metallicity), or IRAS fluxes (affected by the dust abundance and properties) are star formation indicators rather than quantitative measurements (see Gallagher, Bushouse, \& Hunter 1989; Kennicutt 1992). Although it could be argued that near-IR recombination lines such as Br $\gamma$ could be even better measurements of the current SFR, being less affected by extinction than the optical lines, the lack of near-IR instrumentation with a very large field of view makes large-area near-IR surveys impractical at the moment. These considerations imply that the best available way to quantify current star formation in the local universe is by using an $\mathrm{H} \alpha$-selected sample of galaxies. However, it should be kept in mind that if some fraction of newly formed stars is completely hidden by dust, they would not contribute to the 
$\mathrm{H} \alpha$ luminosity, implying that the SFR derived here should be considered a lower limit.

The Universidad Complutense de Madrid (UCM) survey for emission-line objects provides an ideal tool for such a purpose, since galaxies were selected according to their $\mathrm{H} \alpha$ emission. It is a low-dispersion objective-prism survey for low-redshift emission-line galaxies (ELGs), being carried out using the $80 / 120 \mathrm{~cm} \mathrm{f} / 3$ Schmidt telescope at the GermanSpanish Observatory of Calar Alto (Almería, Spain). A $4^{\circ}$ full-aperture prism, yielding a dispersion of $1950 \AA \mathrm{mm}^{-1}$, combined with IIIa-F emulsion, has been used to search for ELGs selected by the presence of $\mathrm{H} \alpha$ emission in their spectra. The instrumental setup is able to record the $\mathrm{H} \alpha+\left[\begin{array}{ll}\mathrm{N} & \text { II }\end{array}\right.$ $\lambda \lambda 6548,6584$ blend in emission for objects up to $z \lesssim 0.045$. The survey itself and the two first discovery lists have been described in detail by Zamorano et al. (1994, 1995). Preliminary results and details are presented in Rego et al. (1989, 1993) and Zamorano et al. (1990, 1992). So far the UCM survey has found 264 candidates in 17 fields covering 471.4 $\mathrm{deg}^{2}$. The overall object density is 0.56 candidates per square degree. More than half of the sample (138 candidates) are galaxies which do not appear in any published catalog. Follow-up imaging and spectroscopy of the survey candidates have been completed and used to confirm the candidates as ELGs and classify them. Morphologically, the UCM survey sample represents a heterogeneous population of galaxies dominated by late-type galaxies ( $66 \%$ being $\mathrm{Sb}$ or later), with $\sim 10 \%$ presenting typical parameters of earlier types and another $\simeq 10 \%$ being irregulars. In all but $22 \%$ of the galaxies we find the emission to come almost entirely from the nuclear regions. Their median luminosity is $\sim 1$ mag fainter than that of magnitude-limited galaxy samples selected in blue plates, with a higher fraction of low-luminosity galaxies. As classified from their spectra and IRAS data, the most commonly found ELGs $(47 \%)$ are intermediate- to low-luminosity objects with a very intense star formation region which dominates the energy output of the galaxy. Their metallicities range from solar values to $1 / 40 Z_{\odot}$, peaking at $1 / 4 Z_{\odot}$. A full analysis has been published in Vitores (1995), Vitores et al. (1995a, b), Gallego (1995), and Gallego et al. (1995a, b). This extensive data set provides the raw materials for the study presented here.

From this catalog, we have built a representative complete sample of star-forming galaxies (which excludes active galactic nuclei [AGNs]) suitable for the statistical study presented in this Letter. In $\S 2$ we discuss the galaxy selection and estimate the $\mathrm{H} \alpha$ luminosity function, which, in $\S 3$, is used to estimate the present-day SFR density. Finally, we summarize our conclusions in $\S 4$.

\section{THE H $\alpha$ LUMINOSITY FUNCTION}

Direct information on the amount and distribution of the present-day SFR in the local universe can be obtained by constructing the LF for galaxies with current star formation activity. The Balmer line emission and, in particular, the $\mathrm{H} \alpha$ luminosity provide a direct signature of the star formation activity occurring in the galaxies. We will now estimate the $\mathrm{H} \alpha$ LF.

In a sample built from an objective-prism survey such as the UCM one, the survey completeness depends primarily on the line flux, not the apparent magnitude. As shown by Salzer (1989), the completeness of a line-selected sample depends on
TABLE 1

H $\alpha$ LUMINOSITY FunCTION FOR THE UCM SURVEY

\begin{tabular}{|c|c|c|}
\hline $\begin{array}{c}\log L(\mathrm{H} \alpha) \\
\left(\operatorname{ergs~s}^{-1}\right)\end{array}$ & $\begin{array}{c}\log \Phi \\
\text { (Number per Mpc }{ }^{3} \\
\text { per } 0.4 \text { interval of } \\
\log L(\mathrm{H} \alpha))\end{array}$ & $\begin{array}{c}\text { Number of } \\
\text { Galaxies }\end{array}$ \\
\hline $40.6 \ldots \ldots \ldots \ldots \ldots$ & -3.380 & 7 \\
\hline $41.0 \ldots \ldots \ldots \ldots \ldots$ & -2.878 & 26 \\
\hline $41.4 \ldots \ldots \ldots \ldots \ldots$ & -3.081 & 43 \\
\hline $41.8 \ldots \ldots \ldots \ldots \ldots$ & -3.323 & 54 \\
\hline $42.2 \ldots \ldots \ldots \ldots \ldots$ & -3.654 & 36 \\
\hline $42.6 \ldots \ldots \ldots \ldots \ldots$ & -4.564 & 10 \\
\hline
\end{tabular}

the emission-line + continuum flux and the contrast between the line and the continuum (the equivalent width of the line). Applying the procedures of Salzer (1989) to the UCM survey sample (Gallego et al. 1995b), we define an arbitrary magnitude scale $m_{\mathrm{L}+\mathrm{C}}$ as a function of the line + continuum flux $F_{\mathrm{L}+\mathrm{C}}\left(\right.$ in $\left.\operatorname{ergs~s}^{-1} \mathrm{~cm}^{-2}\right)$ :

$$
m_{\mathrm{L}+\mathrm{C}}=-17.0-2.5 \log F_{\mathrm{L}+\mathrm{C}} .
$$

A quantitative estimate of the survey completeness can be obtained using the $V / V_{\max }$ test (Schmidt 1968; Huchra \& Sargent 1973). Such a test indicates that all the galaxies in the UCM survey sample with $m_{\mathrm{L}+\mathrm{C}} \leq 17.3$ and $\mathrm{EW}\left(\mathrm{H} \alpha+\left[\begin{array}{ll}\mathrm{N} & \mathrm{II}\end{array}\right)\right.$ $\geq 10 \AA$ have $V / V_{\max } \simeq 0.5$, thus constituting a complete sample (see Gallego et al. 1995b for details). For the remainder of this study, the sample of 176 UCM survey galaxies that fulfill these criteria will be referred to as "the UCM survey representative complete sample." The $m_{\mathrm{L}+\mathrm{C}} \leq 17.3$ limit corresponds to a line + continuum flux of $1.9 \times 10^{-14} \mathrm{ergs} \mathrm{s}^{-1} \mathrm{~cm}^{-2}$.

With such a sample we are able to compute the H $\alpha$ LF. The total $\mathrm{H} \alpha$ luminosity, reddening corrected using the Balmer decrement, has been taken from Gallego et al. (1995a). Distances have been estimated from the galaxy redshifts, assuming a value of $H_{0}=50 \mathrm{~km} \mathrm{~s}^{-1} \mathrm{Mpc}^{-1}$. Note that in considering the object detection procedure, the combined $\mathrm{H} \alpha+[\mathrm{N}$ II $]$ emission has been taken into account, since the objective prism produces very low resolution. However, when $\mathrm{H} \alpha$ luminosities are computed, the [N II] contribution is not included, since the follow-up spectroscopy separates both lines.

If $\Phi[\log L(\mathrm{H} \alpha)]$ is the number of galaxies per unit volume per 0.4 interval in $\log L(\mathrm{H} \alpha)$, then the Schmidt estimator can be written as

$$
\Phi[\log L(\mathrm{H} \alpha)]=\frac{4 \pi}{\Omega} \sum_{i} \frac{1}{V_{\max }^{i}},
$$

where $\Omega$ is the surveyed solid angle and the $V_{\max }^{i}$ represent the volume enclosed by a sphere of radius equal to the maximum distance the galaxy could have and still be detected in the survey. The summation is over all galaxies in the $\mathrm{H} \alpha$ luminosity range $\log L(\mathrm{H} \alpha) \pm 0.5 \Delta \log L(\mathrm{H} \alpha)$. We have used $\Delta \log$ $L(\mathrm{H} \alpha)=0.4$. The LF for the UCM survey galaxies is given in Table 1 .

The $V_{\max }$ method presented here has the advantage of simplicity, but because galaxies are assumed to be distributed homogeneously, the results may be distorted at the faint end if there are local inhomogeneities in the sample. The maximum likelihood estimator described in Mobasher, Sharples, \& Ellis (1993) is independent of such inhomogeneities, provided that the LF has the same shape everywhere. Using this method, we 
obtain a LF that agrees, within the errors, with the one obtained from the $V_{\max }$ method, showing that local inhomogeneities either are not important or average out in the volume sampled by the UCM survey.

Typical $\mathrm{H} \alpha$ luminosities range from $10^{40}$ to $10^{42} \mathrm{ergs} \mathrm{s}^{-1}$. The density of star-forming galaxies decreases with $\mathrm{H} \alpha$ luminosity, as pointed out previously by other authors (Salzer 1989; Boroson, Salzer, \& Trotter 1993). What is more important, however, is that the $\mathrm{H} \alpha$ technique used for the UCM survey recovers a higher number of star-forming galaxies at lower relative levels of star formation activity than surveys carried out in the blue region of the spectrum. This selection effect has clear repercussions when estimating the number and distribution of star-forming galaxies from such blue surveys, since they can miss up to $\sim 50 \%$ of the galaxies undergoing star formation and bias the $\mathrm{H} \alpha$ luminosity distribution (thus the SFR distribution) toward higher luminosities (higher SFRs).

\section{THE SFR DENSITY OF THE LOCAL UNIVERSE}

One of the most important applications of the $\mathrm{H} \alpha$ luminosity function is that it can be used to estimate the current "SFR function" of galaxies, which describes the number of starforming galaxies as a function of their ongoing SFR. Moreover, if an analytical expression is obtained, it can be used to extrapolate observed luminosity density to total luminosity density in the volume considered. The luminosity function for our complete sample of current star-forming galaxies is well fitted by a Schechter (1976) function

$$
\phi(L) d L=\phi^{*}\left(L / L^{*}\right)^{\alpha} \exp \left(-L / L^{*}\right) d\left(L / L^{*}\right)
$$

where the luminosity function $\phi(L)$ is directly related to $\Phi[\log$ $L(\mathrm{H} \alpha)$ ] for $L=L(\mathrm{H} \alpha)$ by the equation

$$
\Phi(\log L) \frac{d \log L}{0.4}=\phi(L) d L
$$

The best-fitting parameters (excluding the first point) are

$$
\begin{aligned}
\alpha & =-1.3 \pm 0.2 \\
\phi^{*} & =10^{-3.2 \pm 0.2} \mathrm{Mpc}^{-3} \\
L^{*} & =10^{42.15 \pm 0.08} \mathrm{ergs} \mathrm{s}^{-1} .
\end{aligned}
$$

Figure 1 shows the binned luminosity distribution, and the solid curve represents the Schechter function with the above parameters.

The errors associated with the derived parameters were obtained using a Monte Carlo method. A large number of simulations were computed with errors in the LF following a Gaussian distribution of $\sigma$ equal to the square root of the number of galaxies in each $\log L(\mathrm{H} \alpha)$ bin. A Schechter function was then fitted to the simulated LFs, yielding a normal distribution for the parameter values. The quoted errors are $1 \sigma$ for these distributions.

If a Schechter function with the above parameters is a good approximation to the luminosity distribution in the volume of universe considered, since $\alpha \leq-2, \phi(L)$ is finite and can be integrated for the whole range of luminosities,

$$
\begin{aligned}
L_{\text {tot }} & =\int_{0}^{\infty} \phi(L) L d L \\
& =\phi^{*} L^{*} \Gamma(2+\alpha) .
\end{aligned}
$$

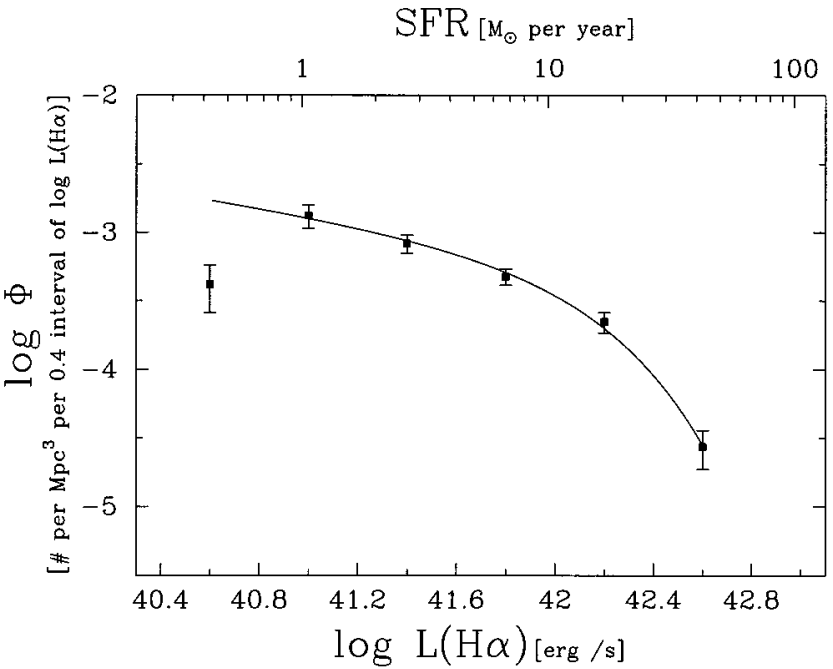

FIG. 1.- $\mathrm{H} \alpha$ luminosity density distribution for a complete sample of UCM survey galaxies. The errors are the square root of the number of galaxies in each bin. The solid line represents the fitted Schechter function to the data points. The scale at the top of the diagram indicates the SFR corresponding to the $\mathrm{H} \alpha$ luminosity (see eq. [6]).

The gamma function $\Gamma(2+\alpha)$ takes the value 1.30 for $\alpha=1.3$, yielding a total $\mathrm{H} \alpha$ luminosity per unit volume of $10^{39.1 \pm 0.2} \mathrm{ergs}$ $\mathrm{s}^{-1} \mathrm{Mpc}^{-3}$ in the local universe $(z \lesssim 0.045)$ for star-forming galaxies with $\mathrm{EW}(\mathrm{H} \alpha+[\mathrm{N}$ II $])>10 \AA$.

We can now relate this $\mathrm{H} \alpha$ luminosity to the SFR following the method introduced by Kennicutt (1983). We use the evolutionary models of Bruzual \& Charlot (1993) to relate the current SFR to the number of ionizing photons produced by the newly formed stars and then use case $\mathrm{B}$ recombination theory to predict the luminosity of the $\mathrm{H} \alpha$ emission line. A Scalo (1986) initial mass function (IMF), including stars in the $0.1 M_{\odot}<M<125 M_{\odot}$ mass range, was used. The transformation can be written as

$$
L(\mathrm{H} \alpha)=9.40 \times 10^{40} \frac{\mathrm{SFR}}{M_{\odot} \mathrm{yr}^{-1}} \operatorname{ergs~s}^{-1} .
$$

The top axis of Figure 1 shows how the $L(\mathrm{H} \alpha)$ scale transforms into a SFR scale using this equation, so that the current SFR function can be directly read from the figure. The total $\mathrm{H} \alpha$ luminosity density translates into a SFR density of $0.013_{-0.005}^{+0.007}$ $\mathrm{M}_{\odot} \mathrm{yr}^{-1} \mathrm{Mpc}^{-3}$, where the main source of uncertainty originates in the normalization factor $\phi^{*}$ of the LF.

Some caution is necessary when interpreting these quantitative results. The $\mathrm{H} \alpha$ luminosity is only sensitive to the SFR in stars with $M \geq 10 M_{\odot}$, the main contributors to the ionizing flux. Thus, the total SFR given here is an extrapolation assuming a reasonable (local neighborhood) IMF. To transform the total SFR values given here into SFRs in stars with $M \geq 10 M_{\odot}$, a factor of 0.044 should be applied. Nevertheless, we have decided to quote total SFRs, since they can provide useful constraints for galaxy formation and evolution models, notwithstanding the IMF uncertainty.

\section{SUMMARY AND CONCLUSIONS}

Using an $\mathrm{H} \alpha$-selected sample of nearby galaxies, we have estimated the $\mathrm{H} \alpha \mathrm{LF}$ for the local universe. We argue that, since the $\mathrm{H} \alpha$ emission provides a good estimate of the ongoing star formation, the galaxies have been selected by their current 
SFR. Therefore, we can use the $\mathrm{H} \alpha \mathrm{LF}$ to determine the SFR function describing the number of star-forming galaxies as a function of their SFR. Integrating over all $\mathrm{H} \alpha$ luminosities (or SFRs), we determine the current SFR density of the local universe for galaxies with $\mathrm{EW}(\mathrm{H} \alpha+[\mathrm{N}$ II]) $>10 \AA$ and $z \lesssim 0.045$. The transformation of $\mathrm{H} \alpha$ luminosities into SFRs depends on the stellar IMF. The values presented in $\$ 3$ correspond to a solar neighborhood Scalo (1986) IMF but can be easily obtained for any IMF by changing equation (6).

We stress that this has been done for the first time for a sample of star-forming galaxies directly selected, as far as it is possible today, by their SFRs. Using $\mathrm{H} \alpha$ as the SFR estimator has clear advantages over optical surveys carried out in the blue and far-IR surveys. It provides a cleaner sample, less biased by metallicity, excitation, and dust properties effects, and covers a very broad range in star formation activity, reaching relatively low levels.

Both the SFR function and the total SFR density determined here can provide direct constraints to galaxy formation and evolution models. They can also play an important role in studies of high-redshift galaxy samples, providing the $z=0$ benchmark to be compared with the numbers of high- $z$ star-forming objects.

We thank S. White, A. G. Vitores, O. Alonso, J. Gorgas, and N. Cardiel for their many valuable comments. This work was supported in part by the Spanish Programa Sectorial de Promoción del Conocimiento under grant PB93-456. A. A. S. acknowledges generous financial support from the Royal Society.

\section{REFERENCES}

Aragón-Salamanca, A. 1995, in ASP Conf. Ser., Fresh Views on Elliptica Galaxies, ed. A. Buzzoni, A. Renzini, \& A. Serrano (San Francisco: ASP), in press

Boroson, T. A., Salzer, J. J., \& Trotter, A. 1993, ApJ, 412, 524

Broadhurst, T. J., Ellis, R. S., \& Glazebrook, K. 1992, Nature, 355, 55

Bruzual, G., \& Charlot, S. 1993, ApJ, 405, 538

Cole, S., Aragón-Salamanca, A., Frenk, C. S., Navarro, J., \& Zepf, E. 1994 MNRAS, 271, 781

Colless, M. 1995, in Wide Field Spectroscopy and the Distant Universe, ed. S. Maddox \& A. Aragón-Salamanca (Singapore: World Scientific), 263

Ellis, R. S., Colless, M., Broadhurst, T. J., Heyl, J., \& Glazebrook, K. 1995, MNRAS, submitted

Gallagher, J. S., Bushouse, H., \& Hunter, D. A. 1989, AJ, 97, 700

Gallagher, J. S., \& Gibson, S. J. 1993, in Panchromatic View of Galaxies, ed. G. Hensler, C. Theis, \& J. S. Gallagher (Gif-sur-Yvette: Editions Frontières), 207

Gallego, J. 1995, PASP, 107, 708

Gallego, J., Zamorano, J., Rego, M., Alonso, O., \& Vitores, A. G. 1995a, A\&AS, in press

Gallego, J., Zamorano, J., Rego, M., \& Vitores, A. G. 1995b, ApJ, submitted Huchra, J. P., \& Sargent, W. L. W. 1973, ApJ, 186, 433

Kauffmann, G., White, S. D. M., \& Guiderdoni, B. 1993, MNRAS, 264, 201

Kennicutt, R. C. 1983, ApJ, 272, 54

- 1992, ApJ, 388, 310
Koo, D. C., \& Kron, R. G. 1992, ARA\&A, 30, 613

Lilly, S. J., Tresse, L., Hammer, F., Crampton, D., \& Le Fevre, O. 1995, ApJ, 455, 108

Mobasher, B., Sharples, R. M., \& Ellis, R. S. 1993, MNRAS, 263, 560

Rego, M., Cordero, M., Zamorano, J., \& Gallego, J. 1993, AJ, 105, 427

Rego, M., Zamorano, J., \& González-Riestra, R. 1989, A\&AS, 79, 443

Salzer, J. J. 1989, ApJ, 347, 152

Scalo, J. M. 1986, Fundam. Cosmic Phys., 11, 1

Schechter, P. 1976, ApJ, 203, 297

Schmidt, M. 1968, ApJ, 151, 393

Songaila, A., Cowie, L. L., Hu, E. M., \& Gardner, J. P. 1994, ApJS, 94, 461

Vitores, A. G. 1995, PASP, 107, 404

Vitores, A. G., Zamorano, J., Rego, M., Alonso, O., \& Gallego, J. 1995a, A\&AS, submitted

. 1995b, A\&A, submitted

Zamorano, J., Gallego, J., Rego, M., Vitores, A. G., \& Alonso, O. 1995, ApJS, submitted

Zamorano, J., Gallego, J., Rego, M., Vitores, A. G., González-Riestra, R., \& Rodríguez-Caderot, G. 1994, ApJS, 95, 387

Zamorano, J., Rego, M., Gallego, J., Vitores, A. G., \& González-Riestra, R. 1992, AJ, 104, 1000

Zamorano, J., Rego, M., González-Riestra, R., \& Rodríguez, G. 1990, A\&AS, 170,353 


\section{ERRATA}

In the Letter "The Current Star Formation Rate of the Local Universe" by J. Gallego, J. Zamorano, A. Aragón-Salamanca, and M. Rego (ApJ, 455, L1 [1995]), the formal errors in the H $\alpha$ luminosity density and the star formation rate (SFR) density for the local universe were calculated assuming that the errors in the Schechter function parameters $\left(\alpha, L^{*}\right.$, and $\left.\phi^{*}\right)$ of the H $\alpha$ luminosity function were independent. Since the errors in these parameters are strongly correlated, the formal errors in the H $\alpha$ luminosity density and the star formation rate density are significantly smaller that those presented in the Letter.

The correct formal errors have now been estimated by computing $L_{\text {tot }}$ (eq. [5]) using the Monte Carlo simulations described in $\S 3$. With the new formal errors, the value of the $\mathrm{H} \alpha$ luminosity density (quoted in the Abstract and in $\S 3$ ) should be $10^{39.09 \pm 0.04}$ ergs $\mathrm{s}^{-1} \mathrm{Mpc}^{-3}$, and the SFR density for the local universe becomes $0.013 \pm 0.001 M_{\odot} \mathrm{yr}^{-1} \mathrm{Mpc}^{-3}$.

Note that although the Schechter function parameters $\alpha, L^{*}$, and $\phi^{*}$ have relatively large uncertainties, the integral of the luminosity function is much more robust, thus having a very small formal error.

In the paper "Magnetocentrifugally Driven Flows from Young Stars and Disks. V. Asymptotic Collimation into Jets" by Frank H. Shu, Joan Najita, Eve C. Ostriker, and Hsien Shang (ApJ, 455, L155 [1995]), Figure 2 contains an error in the displayed values of $C(r)$ and $\theta_{1}(r)$ for the unimportant range $\log _{10} r<1$. The correct version of this figure is reproduced below. This error affects none of the results or conclusions of the paper.

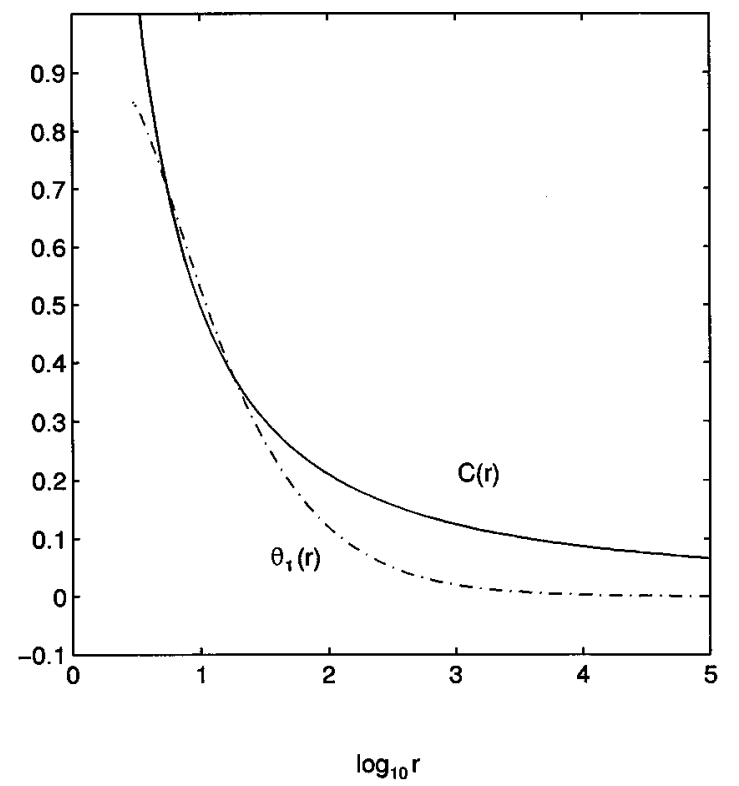

FIG. 2.-Derived values of $C(r)$ and $\theta_{1}(r)$ consistent with the adopted $J(\psi)$ and $\beta(\psi)$ of Fig. 1. 